\title{
Domain state model for exchange bias. I. Theory
}

\author{
U. Nowak and K. D. Usadel \\ Theoretische Tieftemperaturphysik, Gerhard-Mercator-Universität Duisburg, 47048 Duisburg, Germany
}

J. Keller, P. Miltényi, B. Beschoten, and G. Güntherodt

2.Physikalisches Institut, RWTH Aachen, 52056 Aachen, Germany

(Received 10 August 2001; published 17 July 2002)

\begin{abstract}
For a model system consisting of a ferromagnetic layer coupled to a diluted, antiferromagnetic layer extensive Monte Carlo simulations are performed. Exchange bias is observed as a result of a domain state in the antiferromagnetic layer which develops during fiel cooling, carrying an irreversible domain state's magnetization. In agreement with recent experimental observations on $\mathrm{Co} / \mathrm{CoO}$ bilayers a strong dependence of the exchange bias fiel on dilution of the antiferromagnet is found and it is shown that a variety of typical effects associated with exchange bias, such as positive bias, temperature, and time dependencies as well as the dependence on the thickness of the antiferromagnetic layer can be explained within our model.
\end{abstract}

PACS number(s): 75.70.Cn, 75.40.Mg, 75.50.Lk

\section{INTRODUCTION}

When a ferromagnet (FM) is in contact with an antiferromagnet (AFM) a shift of the hysteresis loop along the magnetic-fiel axis can occur which is called exchange bias (EB). Usually, this shift is observed after cooling the entire system in an external magnetic fiel below the Neel temperature $T_{N}$ of the AFM. Although this effect has been well known for many years ${ }^{1,2}$ its microscopic origin is still discussed controversially. For a review of the vast literature on EB the reader is referred to a recent paper by Nogués and Schuller. $^{3}$

In the approach of Malozemoff ${ }^{4-6} \mathrm{~EB}$ is attributed to the formation of domain walls in the AFM below $T_{N}$ perpendicular to the FM/AFM interface due to interface roughness. These domain walls are supposed to occur during cooling in the presence of the magnetized FM and they therefore carry a small net magnetization at the FM/AFM interface. This magnetization is then stabilized towards low temperatures, consequently shifting the hysteresis loop. However, the formation of domain walls in the AFM only due to interface roughness is energetically unfavorable and it has never been proven.

Because of these difficultie other approaches have been developed. In a recent model by $\mathrm{Koon}^{7} \mathrm{~EB}$ is obtained through a mechanism in which a domain wall forms in the AFM parallel to the interface while the magnetization of the FM rotates. This mechanism had been proposed earlier by Mauri et al. ${ }^{8}$ Nevertheless it was shown by Schulthess and Butler $^{9,10}$ that in this model EB vanishes if the motion of the spins in the AFM is not restricted to a plane parallel to the fil as was done in Koon's work. To obtain EB Schulthess and Butler assumed uncompensated AFM spins at the interface. However, their occurrence and stability during a magnetic hysteresis loop is not explained, neither in their model nor in other models ${ }^{11,12}$ although uncompensated AFM spins were observed experimentally. ${ }^{13,14}$

In a previous paper ${ }^{15}$ we have shown both experimentally and by Monte Carlo simulations that it is possible to strongly influenc $\mathrm{EB}$ by replacing magnetic atoms by nonmagnetic ones or by defects (called dilution in the following) not at the FM/AFM interface, but rather throughout the volume part of the AFM. Therefore, in these systems the observed EB is primarily not due to disorder or defects at the interface. Rather, the full antiferromagnetic layer must be involved and we have argued that in our systems EB has its origin in a domain state in the volume part of the AFM which triggers the spin arrangement and the FM/AFM exchange interaction at the interface. This domain state (DS) carries magnetization since it develops during a cooling process in which the AFM is in contact with a saturated FM and eventually also exposed to a magnetic field Dilution favors the formation of this state since then the domain walls can pass preferentially through nonmagnetic sites thus reducing considerably the energy necessary to create a wall.

The DS depends on the concentration of nonmagnetic sites resulting in a dependence of $\mathrm{EB}$ on this concentration. The formation of domains with increasing dilution leads to an increase of the excess magnetization in the AFM and thus to a strong increase of the EB. It is very important to note that the DS is a metastable state which develops and becomes frozen during cooling. Thus it is the result of a dynamical process which requires no further assumptions about structure or size of the AFM domains formed.

Prominent EB systems in which the AFM has a large anisotropy $^{3}$ are $\mathrm{CoO}$, investigated in the experimental part of our work, ${ }^{16}$ or $\mathrm{FeF}_{2}$. We take this as a motivation for modeling the AFM as an Ising system which, from a numerical point of view, is an ideal candidate to study basic properties of EB. The width of the domain walls is zero and the domain size is small so that a significan number of domains fi into a typical system which can be handled numerically. Note, however, that a basic feature of our DS model, i.e., the strong dependence of the EB fiel on dilution, is also obtained for an AFM modeled as a Heisenberg system. ${ }^{17}$

In the present paper we will give a detailed account of our Monte Carlo simulations of the DS model showing that a number of puzzling experimental observations associated with EB is obtained in our model in a straightforward way with a minimal number of assumptions and parameters. The 
paper is organized as follows. In the next section we give a brief review of the physics of domains in diluted Ising antiferromagnets in an external fiel (DAFF). These systems have been studied in great detail in the past and the physics which emerges from these studies is important for understanding EB. In Sec. III our model is described and in Sec. IV our results from Monte Carlo simulations are discussed. For an easier comparison, these results are discussed in the same order as in the experimental part of our work (see the following paper in this issue. ${ }^{16}$ ) Finally, we summarize in the last section.

\section{DOMAINS IN DISORDERED ANTIFERROMAGNETS}

Considerable interest has been focused in recent years on the understanding of magnetic systems with quenched randomness. The diluted Ising antiferromagnet in an external magnetic fiel is an ideal system to study typical properties of structurally disordered systems, as there are domains, metastability, and slow dynamics (for reviews on DAFF see Refs. 18 and 19). Additionally, many of the finding of the DAFF are also relevant for the random-fiel Ising model (RFIM) which has been shown to be in the same universality class. $^{20-22}$

The Hamiltonian of the DAFF can be written as

$$
\mathcal{H}=-J_{\mathrm{AFM}} \sum_{\langle i, j\rangle} \epsilon_{i} \epsilon_{j} \sigma_{i} \sigma_{j}-\mu B \sum_{i} \epsilon_{i} \sigma_{i}
$$

with the antiferromagnetic nearest-neighbor exchange constant $J_{\mathrm{AFM}}<0$ and the magnetic fiel $B$. The $\sigma_{i}= \pm 1$ are normalized Ising spin variables representing spins with an atomic moment $\mu$. A fraction $p$ of sites is left without a magnetic moment $\left(\epsilon_{i}=0\right)$ while the other sites carry a moment $\left(\epsilon_{i}=1\right)$. In simulations, the defect distributions are chosen randomly and an average over different realizations is performed. This models materials where magnetic ions are randomly substituted by nonmagnetic ones, as, for example, in $\mathrm{Fe}_{1-p} \mathrm{Zn}_{p} \mathrm{~F}_{2}$, which is the most prominent DAFF.

Let us firs focus on the phase diagram of the threedimensional DAFF, i.e., on equilibrium properties. In zero fiel the system undergoes a phase transition from the disordered, paramagnetic phase to the long-range-ordered antiferromagnetic phase at the dilution-dependent Néel temperature $T_{N}$ as long as the dilution $p$ is small enough so that the lattice of occupied sites is above the percolation threshold. In the low-temperature region, for small magnetic fields $\mu B$ $\ll J_{\mathrm{AFM}}$, the long-range-ordered phase remains stable in three dimensions ${ }^{23,24}$ while for higher field the DAFF develops a DS, ${ }^{25-27}$ with a spin-glass-like behavior. The reason for the domain formation was originally investigated by Imry and $\mathrm{Ma}$ for the RFIM. ${ }^{28}$ Transferring the so-called Imry-Ma argument to the DAFF, the driving force for the domain formation is a statistical imbalance of the number of impurities of the two antiferromagnetic sublattices within any finite region of the DAFF. This imbalance leads to a net magnetization within that region which couples to the external field $\mathrm{A}$ spin reversal of the region, i.e., the creation of a domain, can hence lower the energy of the system. The necessary energy

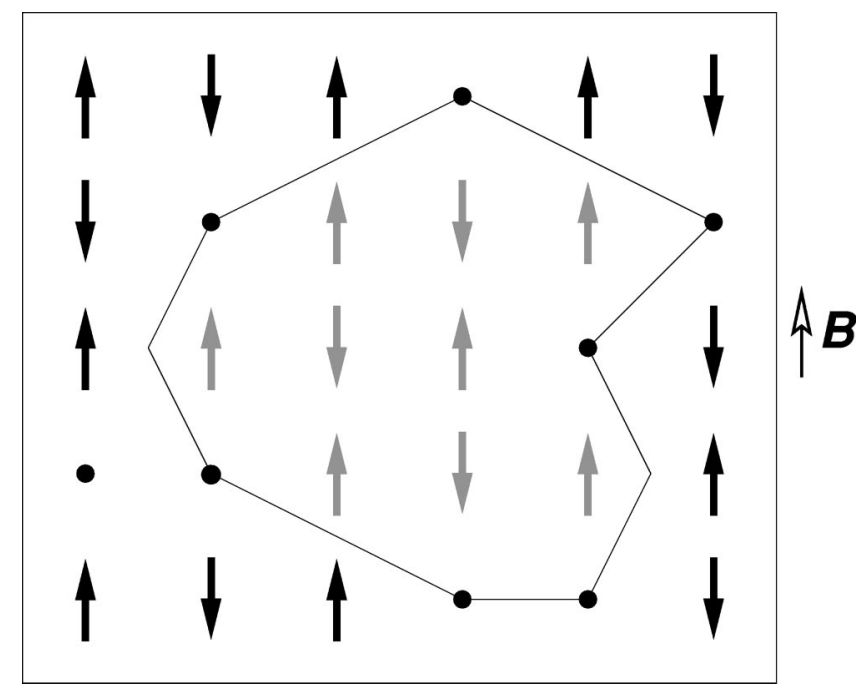

FIG. 1. Schematic illustration of the Imry-Ma argument.

increase due to the formation of a domain wall can be minimized if the domain wall passes preferentially through nonmagnetic defects at a minimum cost of exchange energy. Hence, these domains have nontrivial shapes following from an energy optimization. They have been shown to have a fractal structure with a broad distribution of domain sizes and with scaling laws quantitatively deviating from the original Imry-Ma assumptions. ${ }^{29,30}$

A schematic spin configuratio illustrating the Imry-Ma argument is shown in Fig. 1. The black dots denote defects (nonmagnetic ions or vacancies) and the solid line surrounds a domain in which the staggered magnetization is reversed with respect to the background staggered magnetization outside this domain. The number of uncompensated spins of the domain is three and the number of broken bonds at the domain boundary is five Therefore, for $\mu B>5 / 3\left|J_{\mathrm{AFM}}\right|$ the shown spin configuratio is stabilized by the field

In small field the equilibrium phase of the threedimensional DAFF is long-range ordered. However, if cooled in a fiel $B$ below a certain temperature $T_{i}(B)$, the system usually develops metastable domains. ${ }^{31,32}$ The reason for this metastability is a strong pinning which hinders domain-wall motion. These pinning effects are due to the dilution (random-bond pinning) as well as to the fact that a rough domain wall also carries magnetization in a DAFF (following again the Imry-Ma argument) which couples to the external fiel and hinders domain-wall motion (randomfiel pinning). ${ }^{33}$ Consequently, after cooling the system from the paramagnetic phase within an external field a DAFF freezes in a metastable DS which survives even after switching off the field then leads to a remanent magnetization which decays extremely slowly. ${ }^{34-36}$

The origin of domain-wall pinning is also illustrated in Fig. 1. Consider the case in which the fiel is lowered so that $\mu B$ changes from above to below $5 / 3\left|J_{\mathrm{AFM}}\right|$. Then it is energetically favorable to turn the whole domain. But the corresponding dynamical process will be extremely slow, since the domain wall is pinned at the defects as well as between pairs of spins which are aligned with the field Hence, during 


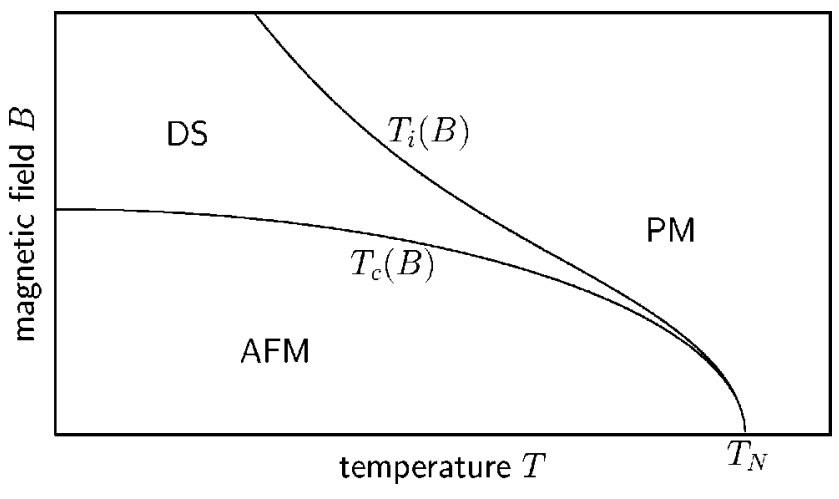

FIG. 2. Schematic phase diagram of a three-dimensional DAFF.

a movement of the domain-wall energy, barriers have to be overcome by thermal activation. This explains why a large domain in general will stay in a metastable state on exponentially long-time scales.

As a consequence, irreversibilities can be observed in a DAFF for $T<T_{i}(B) .{ }^{26,37}$ During fiel cooling from the paramagnetic state, the DAFF develops a DS with a certain surplus magnetization as compared to the long-range-ordered state which has also a finit magnetization due to the response of the system to the field The difference between these two magnetization curves is the irreversible surplus magnetization stemming from the DS of the DAFF.

In Fig. 2 the considerations above are gathered in a schematic phase diagram of the three-dimensional DAFF (see also Refs. 25-27). Shown are the equilibrium phases-longrange ordered (AFM) and paramagnetic - as well as the socalled irreversibility line $T_{i}(B)$. During fiel cooling below this line the system develops a frozen domain state. Note that both the critical temperature $T_{c}$ and the characteristic temperature $T_{i}$ are fiel dependent and that it is $T_{i}(B)>T_{c}(B)$ where both these temperatures approach the Néel temperature for small magnetic field The critical temperature is a decreasing function of the dilution $p$ so that the region of the phase diagram where antiferromagnetic long-range order can occur shrinks with increasing dilution. Below the percolation threshold no long-range order can occur.

In the following we will argue that these well-established properties of the DAFF are the key for understanding EB. During preparation of an EB system, the AFM is cooled (usually) in an external magnetic fiel and additionally under the influenc of an effective interface exchange fiel stemming from the magnetized FM. Hence, the AFM will develop a DS with an irreversible surplus magnetization similar to that of a DAFF after fiel cooling. This irreversible surplus magnetization then controls the EB.

\section{DS MODEL FOR EXCHANGE BIAS}

The Monte Carlo simulations were performed on a model consisting of a FM monolayer exchange coupled to a diluted AFM fil consisting of typically nine monolayers. Only in Sec. IV F do we vary the thickness of the AFM fil studying for a special set of parameters also the thickness dependence of EB. The geometry of the model is sketched in Fig. 3.

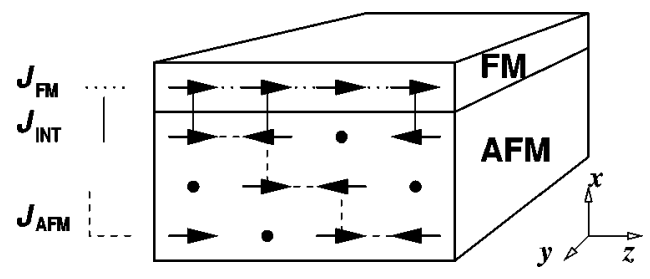

FIG. 3. Sketch of the model with one FM layer and three diluted AFM layers. The dots mark defects.

The FM is described by a classical Heisenberg model with nearest-neighbor exchange constant $J_{\mathrm{FM}}$. We introduce an easy axis in the FM ( $z$ axis, anisotropy constant $d_{z}$ $\left.=0.1 J_{\mathrm{FM}}\right)$ in order to obtain well-define hysteresis loops. $d_{z}$ sets the Stoner-Wohlfarth limit of the coercive field i.e., the zero-temperature limit for magnetization reversal by coherent rotation $\left(\mu B_{c}=2 d_{z}\right.$, in our units, for a fiel parallel to the easy axis). The dipolar interaction is approximated by an additional anisotropy term (anisotropy constant $d_{x}$ $\left.=-0.2 J_{\mathrm{INT}}\right)$ which includes the shape anisotropy, leading to a magnetization which is preferentially in the $y-z$ plane. We checked, however, that its value does not influenc our results.

The AFM is modeled as a magnetically diluted Ising system with an easy axis parallel to that of the FM. Thus the Hamiltonian of our system is given by

$$
\begin{aligned}
\mathcal{H}= & -J_{\mathrm{FM}} \sum_{\langle i, j\rangle \in \mathrm{FM}} \underline{S_{i}} \cdot \underline{S}_{j}-\sum_{i \in \mathrm{FM}}\left(d_{z} S_{i z}^{2}+d_{x} S_{i x}^{2}+\mu \underline{B} \cdot \underline{S_{i}}\right) \\
& -J_{\mathrm{AFM}} \sum_{\langle i, j\rangle \in \mathrm{AFM}} \epsilon_{i} \epsilon_{j} \sigma_{i} \sigma_{j}-\sum_{i \in \mathrm{AFM}} \mu B_{z} \epsilon_{i} \sigma_{i} \\
& -J_{\mathrm{INT}} \sum_{\langle i \in \mathrm{AFM}, j \in \mathrm{FM}\rangle} \epsilon_{i} \sigma_{i} S_{j z} .
\end{aligned}
$$

The firs line contains the energy contribution of the FM. The second line describes the diluted AFM and is identical to Eq. (1). The third line includes the exchange coupling across the interface between FM and AFM, where it is assumed that the Ising spins in the topmost layer of the AFM interact with the $z$ component of the Heisenberg spins of the FM. For the nearest-neighbor exchange constant $J_{\mathrm{AFM}}$ of the AFM which mainly determines its Néel temperature we set $J_{\mathrm{AFM}}$ $=-J_{\mathrm{FM}} / 2$. For simplicity, we assume the same absolute value for the coupling constant $\left(J_{\mathrm{INT}}= \pm J_{\mathrm{AFM}}\right)$ as for the AFM leaving the sign open for later discussion. Also, we assume that the values of the magnetic moments of FM and AFM are identical $(\mu)$. In the experimental part of our work $^{16}$ the structure and dilution of the AFM interface layer is held fixed We do the same in the simulations and fi the interface monolayer of the AFM at a dilution of $50 \%$ and vary only the dilution $p$ in the volume of the AFM.

Equation (2) suggests a simple ground-state argument for the strength of the bias field Assuming that all spins in the FM remain parallel during fiel reversal and that some net magnetization of the interface layer of the AFM remains constant during the reversal of the FM a simple calculation gives the usual estimate for the bias field 


$$
l \mu B_{\mathrm{EB}}=J_{\mathrm{INT}} m_{\mathrm{INT}},
$$

where $l$ is the number of FM layers (in our simulations always $l=1)$ and $m_{\mathrm{INT}}$ is the interface magnetization of the AFM per spin. For an ideal uncompensated interface one would expect $m_{\mathrm{INT}}=1$ which leads to a much too high bias field while for an ideal compensated interface, on the other hand, one would expect $m_{\mathrm{INT}}=0$ and, hence, $B_{\mathrm{EB}}=0$. Experimentally, however, often there is on the one hand no big difference between compensated and uncompensated interfaces and on the other hand it is found that $B_{\mathrm{EB}}$ is much smaller than $J_{\mathrm{INT}} / l \mu$. The solution of this puzzle is, as will be shown in the following, that $m_{\mathrm{INT}}$ is neither constant during fiel reversal nor is it a simple known quantity.

\section{MONTE CARLO SIMULATIONS}

We use Monte Carlo methods with a heat-bath algorithm and single-spin-fli methods for the simulation of the model explained above. The trial step of the spin update is a small variation around the initial spin for the Heisenberg model and - as usual-a spin fli for the Ising model. ${ }^{38}$ We perform typically 40000 Monte Carlo steps for a complete hysteresis loop.

Since we are not interested in the critical behavior of the DS model, we do not perform a systematic finite-siz analysis. To observe the domain structure of the AFM we have to guarantee that typical length scales of the domain structure fi into our system. Therefore, we show here only results for rather large systems of lateral extension $L \times L$ with $L=128$ and we used periodic boundary conditions within the fil plane. Nevertheless, we also varied the lateral system size $L$ and checked that there are no relevant finite-siz effects as long as the system is not much smaller.

The main quantities which we monitor are the thermal averages of the $z$ component of the magnetic moment for each individual monolayer normalized to the magnetic moment of the saturated monolayer. In the following we will use reduced field $b=\mu B /\left|J_{\mathrm{INT}}\right|$.

\section{A. Hysteresis}

In our simulations the system is cooled from above to below the ordering temperature of the AFM. During cooling the FM is initially magnetized along the easy $z$ axis resulting in a nearly constant exchange fiel for the AFM monolayer at the interface. The system is either cooled in zero fiel or in the presence of an external magnetic fiel $\underline{b}_{\mathrm{FC}}=b \hat{z}$, called cooling fiel in the following. In addition to the exchange fiel mentioned above this fiel then acts also on the volume part of the AFM. Both cooling mechanisms are discussed in the following. When the desired fina temperature is reached a magnetic fiel $b=b \underline{\hat{z}}+b_{y} \hat{\underline{y}}$ is applied which also has a small, in all simulations, fixe perpendicular fiel component $b_{y}=0.1$ in order to defin a certain path for the rotation of the magnetization during fiel reversal and to avoid the system becoming trapped in a metastable state. The $z$ component of the field $b$, is then reduced in steps of $\Delta b=0.008$
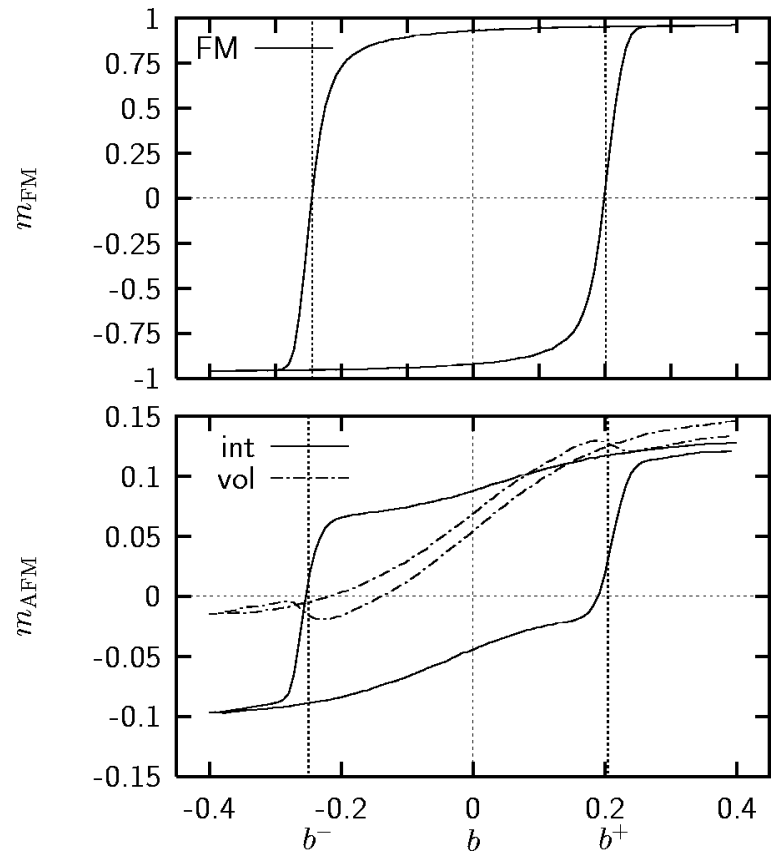

FIG. 4. Simulated hysteresis loops of the DS model explained in the text for $p=0.5$ and $k_{B} T=0.2 J_{\text {INT }}$ with positive interface coupling, $J_{\mathrm{INT}}=\left|J_{\mathrm{AFM}}\right|$. The fiel during cooling was 0.4. Shown is the magnetic moment of the FM, the interface monolayer of the AFM, and the volume part of the AFM.

down to a certain minimum value and afterwards raised again to the initial value. This corresponds to one cycle of the hysteresis loop.

Typical hysteresis loops are depicted in Fig. 4 for a cooling fiel $b_{\mathrm{FC}}=0.4$ assuming a positive interface coupling. Shown are results for the magnetization of the FM (upper figur ) as well as that of the AFM interface monolayer and the sum of all the other AFM layers. For the latter we use in the following the term volume part of the AFM only in order to distinguish between interface and volume contributions of the AFM.

An EB is observed clearly and we determine the corresponding EB fiel as $b_{\mathrm{EB}}=\left(b^{+}+b^{-}\right) / 2$ where $b^{+}$and $b^{-}$ are those field of the hysteresis loop branches for increasing and decreasing field where the easy axis component of the magnetization of the FM becomes zero.

The magnetization curve of the volume part of the AFM is shifted upwards due to the fact that after fiel cooling the AFM is in a DS with a surplus magnetization. The same is true also for the interface layer of the AFM, which during cooling experienced the external fiel in addition to the exchange fiel of the FM, both having the same direction. This shifted interface magnetization of the AFM acts as an additional effective fiel on the FM, resulting in EB. Note that the interface of the AFM clearly shows a hysteresis, following the coupling to the FM.

For comparison we show in Fig. 5 hysteresis loops for a negative interface coupling. This system was cooled in zero fiel but it also shows an EB due to the interaction of the AFM with the saturated FM during the cooling procedure. An important observation is that the interface magnetization 

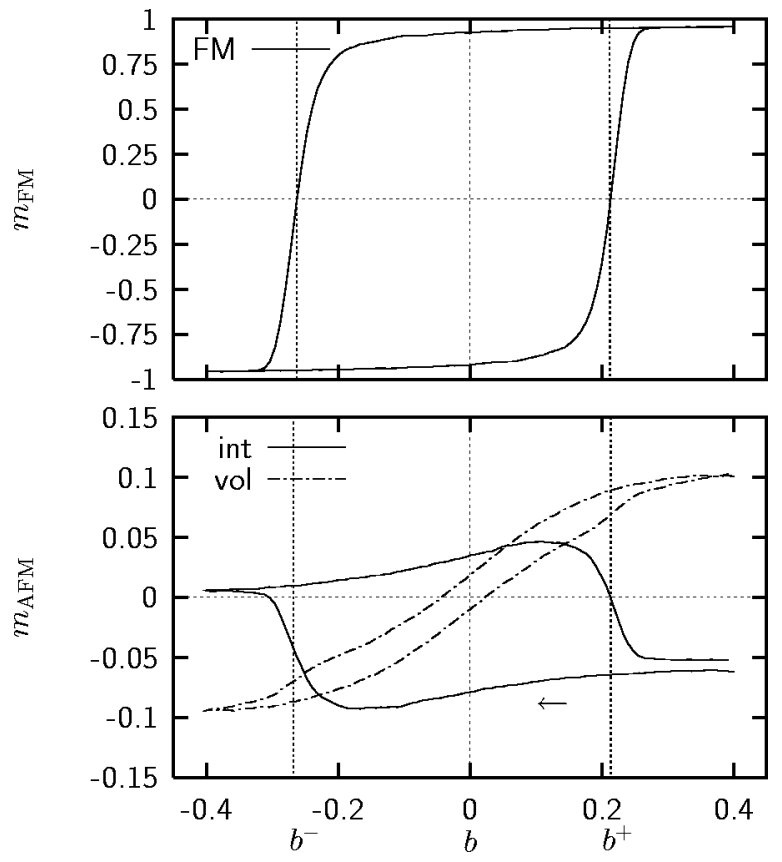

FIG. 5. Simulated hysteresis loops as in Fig. 4, but after cooling in zero fiel and with negative interface coupling $J_{\mathrm{INT}}=J_{\mathrm{AFM}}$.

of the AFM is now shifted downwards due to the fact that the exchange coupling to the FM is negative. Again, this shifted interface magnetization of the AFM acts as an additional effective fiel on the FM resulting in EB. The curve of the volume part of the AFM is not shifted after zero-fiel cooling.

For future reference we defin the quantity $m_{\text {IDS }}$ $=m_{\mathrm{AFM}}\left(b^{+}\right)+m_{\mathrm{AFM}}\left(b^{-}\right)$which characterizes the vertical shift of the hysteresis loop of the AFM interface layer. Here $m_{\mathrm{AFM}}\left(b^{+}\right)$denotes the magnetization of the AFM at $b^{+}$for increasing fiel and similarly for $m_{\mathrm{AFM}}\left(b^{-}\right)$. This quantity, $m_{\mathrm{IDS}}$, measures the irreversible domain state magnetization of the AFM interface layer which is responsible for the EB fiel and it is precisely this quantity which has to be inserted in the right-hand side of Eq. (3) instead of an usually assumed constant $m_{\mathrm{AFM}}$, yielding

$$
l \mu B_{\mathrm{EB}}=J_{\mathrm{INT}} m_{\mathrm{IDS}} .
$$

A detailed, quantitative comparison of this equation with simulation results is given later in Fig. 11.

In experiments usually the magnetization of the whole FM/AFM bilayer is measured which is just the sum of the three curves shown in Figs. 4 and 5, respectively. The corresponding sample magnetization loop might not only be shifted horizontally but also vertically. The vertical shift contains contributions from the volume part of the AFM as well as from its interface.

The volume magnetization of the AFM is induced by the applied fiel and hence not shifted when the cooling fiel is zero (see Fig. 5) and shifted upwards when it is finit (Fig. 4). The interface contribution depends on the sign of the interface coupling. ${ }^{39}$ Our considerations are also confirme in the experimental part of this work ${ }^{16}$ where an analysis of

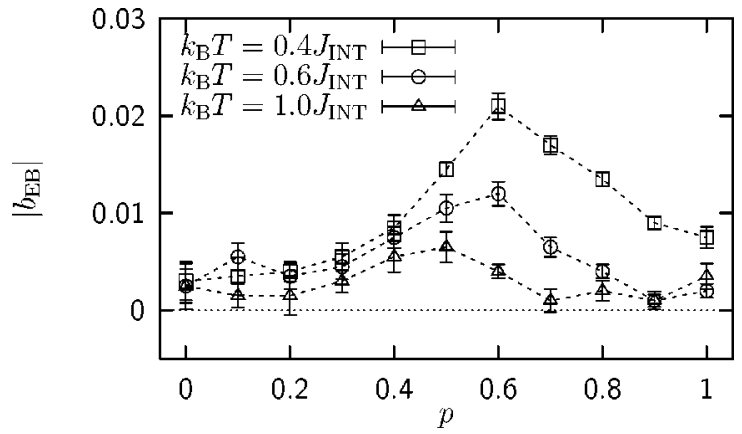

FIG. 6. EB fiel as a function of the dilution $p$ of the AFM for $k_{B} T=0.4,0.6$, and $1.0 J_{\mathrm{INT}}$.

the vertical shift of hysteresis loops is performed leading to the conclusion that in our $\mathrm{Co} / \mathrm{CoO}$ systems the interface coupling is positive.

\section{B. Influenc of dilution}

An important observation in our previous paper ${ }^{15}$ was the strong dependence of the EB on dilution of the AFM. For a further investigation of this effect we vary the volume part dilution $p$ of the AFM keeping the dilution of the interface layer fixed The system is cooled in zero magnetic field A configurationa average is performed over eight different defect realizations leading to a significan reduction of the statistical errors and to much smoother curves in Fig. 6 as compared to results published previously. ${ }^{15}$

In agreement with experimental finding ${ }^{15,16}$ we observe a strong dependence of the EB on dilution. Since dilution favors the formation of domains it leads to an increase of the magnetization in the AFM and thus to a strong increase of the EB upon dilution. For larger dilution, on the other hand, EB drops which is due to a loss of connectivity of the AFM spin lattice and a corresponding decrease of the Néel temperature. Upon dilution of the AFM, isolated spin clusters appear which do not contribute to an AFM net magnetization on longer-time scales leading to a decrease of EB for very high dilution.

We would like to emphasize that the maximum strength of the simulated EB is up to $0.02 \mu B / J_{\text {INT }}$. For a comparison with the corresponding experimental findings ${ }^{15,16}$ we use Eq. (4) with $l=$ nine layers, $J_{\mathrm{INT}}=-3.2 \times 10^{-22} \mathrm{~J}$ and $\mu$ $=1.7 \mu_{B}$ which leads to bias fiel values of up to $30 \mathrm{mT}$, a value which is smaller than found experimentally ${ }^{16}$ but nevertheless is of the correct order of magnitude.

For very small dilution the DS model shows only a very small EB. Although the interface AFM monolayer has a 50\% dilution, domains are practically not formed in the AFM for very small dilution because a domain in the volume part of the AFM would require breaking AFM bonds which costs too much energy. On the other hand, in the experiments on $\mathrm{Co} / \mathrm{CoO},{ }^{15,16} \mathrm{~EB}$ is also observed for zero dilution. This can be explained by other imperfections such as e.g., grain boundaries in the twinned AFM layer which similar to dilution also reduce the domain-wall energy, thus leading to domain formation and EB even without dilution of the AFM bulk. 

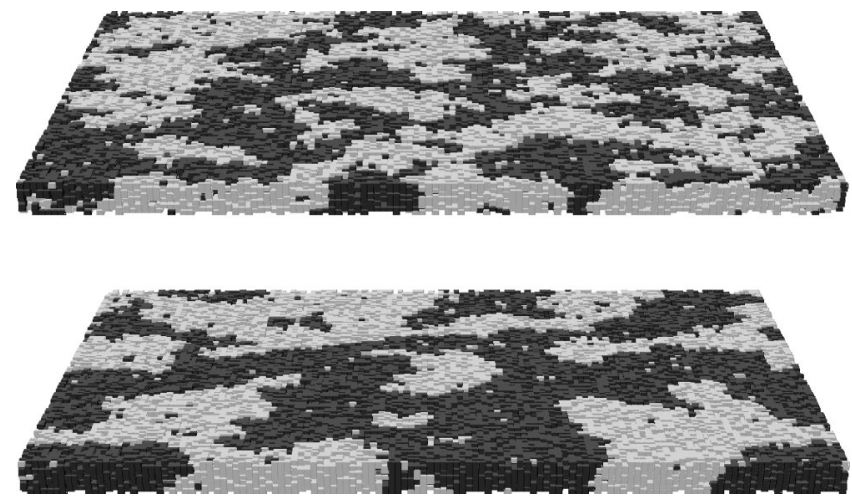

FIG. 7. Frozen domain states of the AFM. Shown are staggered spin configuration of the AFM after the initial cooling procedure for dilution $p=0.5$ (above) and $p=0.3$ (below).

Two domain states formed in the AFM for different values of the dilution are shown in Fig. 7. The fractal structure of these domains is obvious. It has been observed previously in bulk systems and analyzed in detail. ${ }^{29,30}$ The structure of the domains observed depends on dilution; the smaller the dilution, the coarser the domains. Note that there is only little variation of this structure perpendicular to the film especially for the less diluted sample. The importance of AFM domains for the EB effect was also demonstrated in recent spectroscopy measurements. ${ }^{40,41}$

\section{Temperature dependence}

The temperature dependence of the EB fiel is shown in Fig. 8 as an example for a fixe AFM dilution of $p=0.5$. In agreement with experiments ${ }^{15,16}$ the fiel decreases with temperature almost linearly going to zero at a temperature $T_{B}$ of the order of the Neel temperature. $T_{B}$ is called the blocking temperature. It is important to note that $T_{B}$ is not equal to the Néel temperature of the bulk AFM for several reasons.

Reconsidering the phase diagram of the DAFF (Fig. 2), the frozen DS of the AFM occurs after cooling in a fiel below the irreversibility temperature $T_{i}(b)$. Within this interpretation, the blocking temperature corresponds to $T_{i}(b)$ where in an EB system the role of the cooling fiel is complemented (or replaced) by the interface exchange fiel of the FM. Since it is always $T_{i}(b)<T_{N}$ the blocking tem-

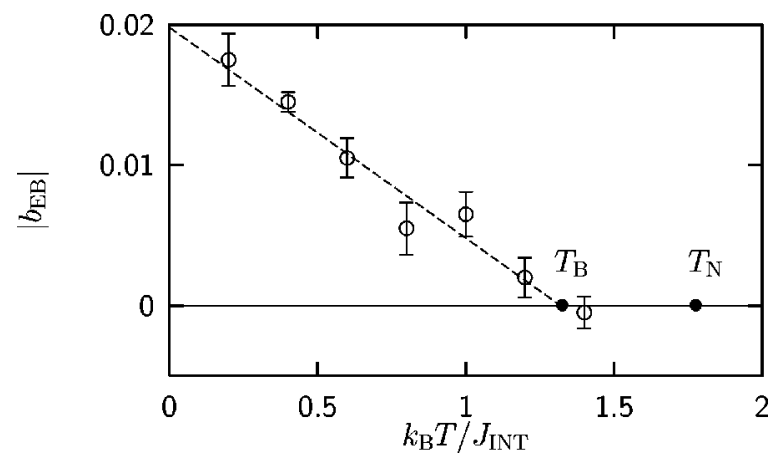

FIG. 8. Temperature dependence of the EB fiel for $p=0.5$. The line is a guide to the eye. The Néel temperature $T_{N}$ of a $50 \%$ diluted bulk AFM is approximately $1.8 J_{\mathrm{INT}} / k_{B}$ (Refs. 26 and 27).



FIG. 9. Training effect: dependence of EB fiel on the number of consecutive hysteresis cycles. $k_{B} T=0.6 J_{\mathrm{INT}}, p=0.5$. The dotted line is a guide to the eye.

perature should be (at least slightly) below the Néel temperature and should be dependent on the strength of the interface exchange field Note also that the Néel temperature depends besides dilution (see also Ref. 16) also on the thickness of the AFM layer and for a very thin layer it may differ considerably from that of an extended three-dimensional system.

\section{Training effect}

An important property of the dynamics of the DAFF is the slow relaxation of the remanent magnetization, i.e., the magnetization obtained after switching off the cooling field Here it is known that the remanent magnetization of the DS relaxes nonexponentially on extremely long-time scales after the fiel is switched off $\mathrm{f}^{3-36}$ or even within the applied field ${ }^{33,37,42}$ In the DS model EB is related to this remanent magnetization. This implies a decrease of EB due to slow relaxation of the AFM DS.

As an example for the relaxation of a DS, Fig. 9 shows the so-called training effect, i.e., the decrease of the EB fiel with perpetual repetition of fiel cycles. As in the corresponding experiments, ${ }^{16}$ after a sharp decrease the EB fiel remains nearly constant.

The reason for the training effect can be understood further from Figs. 4 and 5, where it is shown that the hysteresis loop of the AFM interface layer is not closed on the righthand side. This implies that the DS magnetization is lost partly during the hysteresis loop due to a rearrangement of the AFM domain structure. This loss of magnetization clearly leads to a reduction of the EB.

\section{E. Cooling fiel dependence}

Positive EB (Ref. 43) or reduced EB (Ref. 44) is observed experimentally in certain systems after cooling in strong magnetic fields As we will show in the following, the occurrence of positive bias depends on the sign of the exchange coupling between AFM and FM layers.

Suppose this coupling is positive. If the system is cooled with the FM in a magnetized state and with zero applied fiel the induced magnetization in the AFM after cooling below the Neel temperature is parallel to that of the FM. The EB is negative and its absolute value increases slightly if an external fiel is applied parallel to the FM magnetization during 


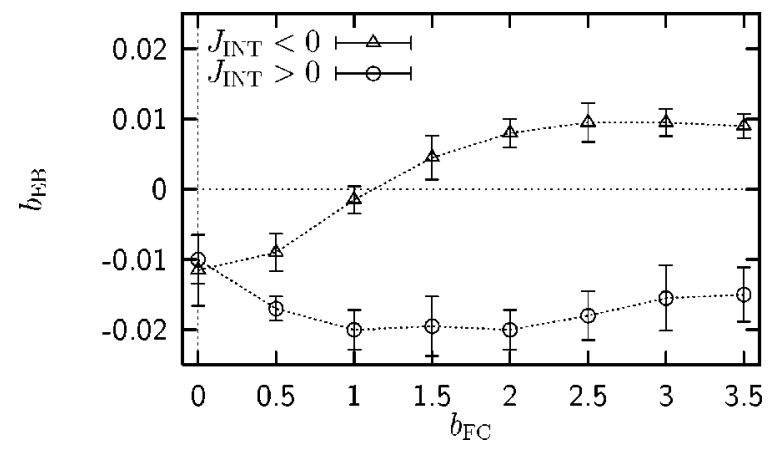

FIG. 10. EB fiel versus strength of the magnetic fiel during the initial cooling procedure for negative and positive interface coupling. $k_{B} T=0.2 J_{\mathrm{INT}}, p=0.5$.

the cooling procedure favoring the same direction of the remanent magnetization of the AFM. This is shown in the lower curve in Fig. 10. This curve corresponds to Fig. 11 in the experimental part of our work, ${ }^{16}$ which confirm that in our $\mathrm{Co} / \mathrm{CoO}$ systems the interface coupling is indeed positive.

If, on the other hand, the exchange coupling is negative, the irreversible DS magnetization in the AFM when cooled in zero applied fiel is opposite to the FM magnetization. When cooled in an external fiel this fiel and the action of the FM on the AFM compete with each other. For small enough external fiel this results in a reduction of the EB field But during cooling in a strong fiel the latter overrules the exchange fiel of the FM and as a consequence the AFM forms domains with the surplus magnetization being parallel to the external field and also to the magnetization of the FM. This then results in a positive EB as shown in the upper curve of Fig. 10. Note that the change of the sign of the EB occurs exactly when the fiel during cooling exceeds the absolute value of the interface exchange constant $(b=1$ in our units) providing the FM is saturated. Hence, positive bias occurs only in systems with negative interface coupling and when it is possible to reach magnetic-fiel values larger than the interface coupling. These results are in agreement with recent experimental finding obtained for $\mathrm{Fe} / \mathrm{FeF}_{2}$ and $\mathrm{Fe} / \mathrm{MnFe}_{2}$ bilayers. $^{39}$

Our explanation for positive $\mathrm{EB}$ - that a negative interface magnetization of the AFM can be overruled by the cool-

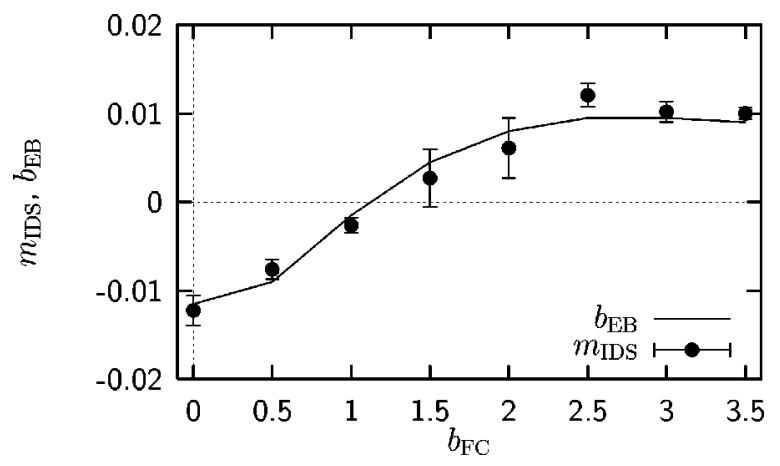

FIG. 11. Comparison of the EB fiel for negative interface coupling (from Fig. 10) with the irreversible DS magnetization of the AFM interface layer.

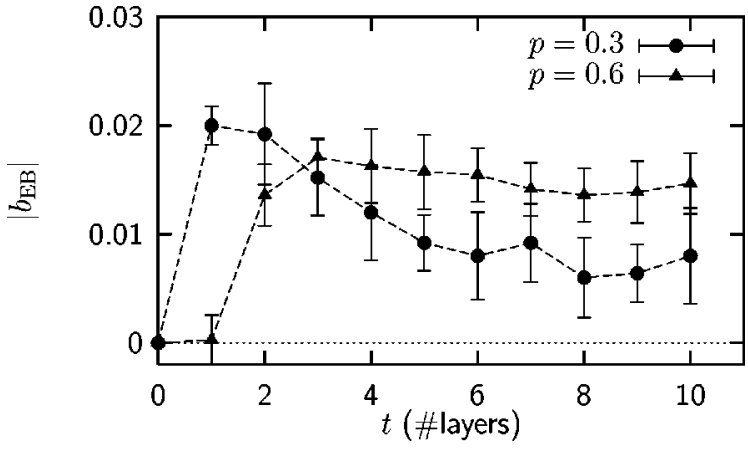

FIG. 12. EB fiel versus thickness of the AFM layer.

ing field- $\mathrm{i}$ further confirme by a direct analysis of the interface magnetization of the AFM shown in Fig. 11. Here we compare our EB fiel from Fig. 10 for negative $J_{\text {INT }}$ directly with the irreversible DS magnetization, $m_{\text {IDS }}$, of the AFM interface layer as define earlier in Sec. IV A. $m_{\text {IDS }}$ coincides nearly exactly with the EB field - result which also confirm Eq. (4). Note, that we use reduced fields $b_{\mathrm{EB}}$ $=\mu B_{\mathrm{EB}} /\left|J_{\mathrm{INT}}\right|$, and that it is $l=1$ since we have one FM monolayer.

\section{F. Thickness dependence}

Experimentally it has been found that the EB increases with the thickness $t$ of the AFM fil for thin films For larger thicknesses the bias fiel often approaches a constant value or it even decreases (see Ref. 16, and references therein). We have studied the thickness dependence of EB for an AFM with dilutions $p=0.3$ and $p=0.6$, respectively. The system is cooled from above to below the ordering temperature of the AFM in an applied external cooling fiel $b_{c}=0.5$ and we assume ferromagnetic interface coupling, $J_{\mathrm{INT}}=-J_{\mathrm{AFM}}$. Our simulation results are shown in Fig. 12.

For smaller dilution $(p=0.3)$ the EB fiel goes over a maximum and then decreases monotonically with increasing $t$. Our explanation for the decrease of EB is that with increasing $t$ it becomes more and more difficul to form domain walls which, as can be seen from Fig. 7, are perpendicular to the interface extending through the whole AFM layer. The corresponding domain-wall energy increases with $t$ but it can be reduced by reducing the number of domain walls, i.e., by the formation of larger domains which then reduce the bias fiel (see also Fig. 2 in Ref. 45).

The increase of the domain-wall energy with increasing AFM thickness is much less pronounced for higher dilution $(p=0.6)$ and therefore it is even possible that the bias fiel becomes practically constant for large thicknesses. The rapid increase for very thin film can be explained by the fact that here the domains are not sufficientl stable to keep their DS magnetization during the hysteresis. Hence, for an EB one needs a minimal AFM layer thickness. A more detailed investigation of the thickness dependence of the bias fiel has been published elsewhere both experimentally ${ }^{46}$ and theoretically. ${ }^{45}$ 


\section{CONCLUSIONS}

In conclusion, we have shown, both experimentally ${ }^{16}$ and by Monte Carlo simulations, that diluting the AFM in the volume part away from the FM/AFM interface significantl enhances EB. This dilution supports the formation of domains in the volume of the AFM which carry magnetization. The DS of the AFM is to a large extent frozen during hysteresis. The irreversible part of the DS magnetization at the AFM interface leads to EB.

In this type of model which we consider here, domain formation is crucial for the existence of EB. Without domain formation there would be no $\mathrm{EB}$ for compensated interfaces and a much too high EB for uncompensated interfaces. Defects in the AFM favor domain formation and thus make the distinction between compensated and uncompensated interfaces to a large extent obsolete. Also, it should be noted that the occurrence of a DS with an irreversible surplus magnetization is not restricted to diluted AFM's. Spin glasses, for instance, show similar features and it is known that the EB effect occurs also in compounds of FM and spin glass. ${ }^{3}$ In these systems we believe the same mechanism leading to EB in our DS model is also at work.

Important features of EB systems found experimentally have their counterpart in the simulations, such as the order of magnitude of EB fields the shape of hysteresis curves, the dilution dependence of EB, its temperature dependence, the training effect, the occurrence of positive EB, and the dependence of EB on AFM thickness. The DS model considered in this work is designed for systems in which the AFM has a strong uniaxial anisotropy so that it can be approximated as an Ising system. For smaller anisotropies a Heisenberg model is more appropriate. Very recently we have shown that the basic feature of the DS model, namely, domain formation in the bulk of the AFM, is not restricted to an AFM with strong anisotropy. ${ }^{17}$

In conclusion, our simulations strongly suggest that the existence of a DS in the AFM, enhanced by defects or any other mechanism reducing the energy necessary to form domains in the volume part of the AFM, is a common feature of FM/AFM compounds showing a significan EB.

\section{ACKNOWLEDGMENTS}

We thank A. Misra for providing us with the simulation data shown in Fig. 12. This work has been supported by the Deutsche Forschungsgemeinschaft through SFB Grant Nos. 341 and 491.
${ }^{1}$ W.H. Meiklejohn and C.P. Bean, Phys. Rev. 102, 1413 (1956).

${ }^{2}$ W.H. Meiklejohn and C.P. Bean, Phys. Rev. 105, 904 (1957).

${ }^{3}$ J. Nogués and I.K. Schuller, J. Magn. Magn. Mater. 192, 203 (1999).

${ }^{4}$ A.P. Malozemoff, Phys. Rev. B 35, 3679 (1987).

${ }^{5}$ A.P. Malozemoff, J. Appl. Phys. 63, 3874 (1988).

${ }^{6}$ A.P. Malozemoff, Phys. Rev. B 37, 7673 (1988).

${ }^{7}$ N.C. Koon, Phys. Rev. Lett. 78, 4865 (1998).

${ }^{8}$ D. Mauri, H.C. Siegmann, P.S. Bagus, and E. Kay, J. Appl. Phys. 62, 3047 (1987).

${ }^{9}$ T.C. Schulthess and W.H. Butler, Phys. Rev. Lett. 81, 4516 (1998).

${ }^{10}$ T.C. Schulthess and W.H. Butler, J. Appl. Phys. 85, 5510 (1999).

${ }^{11}$ M.D. Stiles and R.D. McMichael, Phys. Rev. B 59, 3722 (1999).

${ }^{12}$ M. Kiwi, J. Mejía-López, R.D. Portugal, and R. Ramírez, Europhys. Lett. 48, 573 (1997).

${ }^{13}$ K. Takano, R.H. Kodama, A.E. Berkowitz, W. Cao, and G. Thomas, Phys. Rev. Lett. 79, 1130 (1997).

${ }^{14}$ K. Takano, R.H. Kodama, A.E. Berkowitz, W. Cao, and G. Thomas, J. Appl. Phys. 83, 6888 (1998).

${ }^{15}$ P. Miltényi, M. Gierlings, J. Keller, B. Beschoten, G. Güntherodt, U. Nowak, and K.D. Usadel, Phys. Rev. Lett. 84, 4224 (2000).

${ }^{16}$ J. Keller, P. Miltényi, B. Beschoten, G. Güntherodt, U. Nowak, and K.D. Usadel, following paper, Phys. Rev. B 66, 014431 (2002).

${ }^{17}$ U. Nowak, A. Misra, and K.D. Usadel, J. Magn. Magn. Mater. 240, 249 (2002).

${ }^{18}$ W. Kleemann, Int. J. Mod. Phys. B 7, 2469 (1993).

${ }^{19}$ D. P. Belanger, in Spin Glasses and Random Fields, edited by A. P. Young (World Scientific Singapore, 1998).

${ }^{20}$ S. Fishman and A. Aharony, J. Phys. C 12, L729 (1979).
${ }^{21}$ J.L. Cardy, Phys. Rev. B 29, 505 (1984).

${ }^{22}$ A. Hartmann and U. Nowak, Eur. Phys. J. B 7, 105 (1999).

${ }^{23}$ J.Z. Imbrie, Phys. Rev. Lett. 53, 1747 (1984).

${ }^{24}$ J. Bricmont and A. Kupiainen, Phys. Rev. Lett. 59, 1829 (1987).

${ }^{25}$ F.C. Montenegro, A.R. King, V. Jaccarino, S.-J. Han, and D.P. Belanger, Phys. Rev. B 44, 2155 (1991).

${ }^{26}$ U. Nowak and K.D. Usadel, Phys. Rev. B 44, 7426 (1991).

${ }^{27}$ K.D. Usadel and U. Nowak, J. Magn. Magn. Mater. 104-107, 179 (1992).

${ }^{28}$ Y. Imry and S. Ma, Phys. Rev. Lett. 35, 1399 (1975).

${ }^{29}$ U. Nowak and K.D. Usadel, Phys. Rev. B 46, 8329 (1992).

${ }^{30}$ J. Esser, U. Nowak, and K.D. Usadel, Phys. Rev. B 55, 5866 (1997).

${ }^{31}$ R.J. Birgeneau, R.A. Cowley, G. Shirane, and H. Yoshizawa, J. Stat. Phys. 34, 817 (1984).

${ }^{32}$ D.P. Belanger, M. Rezende, A.R. King, and V. Jaccarino, J. Appl. Phys. 57, 3294 (1985).

${ }^{33}$ J. Villain, Phys. Rev. Lett. 52, 1543 (1984).

${ }^{34}$ S.-J. Han, D.P. Belanger, W. Kleemann, and U. Nowak, Phys. Rev. B 45, 9728 (1992).

${ }^{35}$ U. Nowak, J. Esser, and K.D. Usadel, Physica A 232, 40 (1996).

${ }^{36}$ M. Staats, U. Nowak, and K.D. Usadel, Phase Transitions 65, 159 (1998).

${ }^{37}$ P. Pollak, W. Kleemann, and D.P. Belanger, Phys. Rev. B 38, 4773 (1988).

${ }^{38}$ U. Nowak, in Annual Reviews of Computational Physics IX, edited by D. Stauffer (World Scientific Singapore, 2000), p. 105.

${ }^{39}$ J. Nogués, C. Leighton, and I.K. Schuller, Phys. Rev. B 61, 1315 (2000).

${ }^{40}$ F. Nolting, A. Scholl, J. Stöhr, J.W. Seo, J. Fompeyrine, H. Siegwart, J.-P. Locquet, S. Anders, J. Lüning, E.E. Fullerton, M.F. 
Toney, M.R. Scheinfein, and H.A. Padmore, Nature (London) 405, 767 (2000).

${ }^{41}$ H. Ohldag, A. Scholl, F. Nolting, S. Anders, F.U. Hillebrecht, and J. Stöhr, Phys. Rev. Lett. 86, 3419 (2001).

${ }^{42}$ U. Nowak and K.D. Usadel, Phys. Rev. B 39, 2516 (1989).

${ }^{43}$ J. Nogués, D. Lederman, T.J. Moran, and I.K. Schuller, Phys. Rev. Lett. 76, 4624 (1996).
${ }^{44}$ T.J. Moran, J.M. Gallego, and I.K. Schuller, J. Appl. Phys. 79, 5109 (1996).

${ }^{45}$ U. Nowak, A. Misra, and K.D. Usadel, J. Appl. Phys. 89, 7269 (2001).

${ }^{46}$ B. Beschoten, P. Miltényi, J. Keller, and G. Güntherodt, J. Magn. Magn. Mater. 240, 248 (2002). 